\title{
An Advanced Impedance Calibration Method for Nanoscale Microwave Imaging at Broad Frequency Range
}

\author{
M. Kasper, G. Gramse, and F. Kienberger
}

\begin{abstract}
A new calibration method for nanoscale complex impedance imaging with the scanning microwave microscope (SMM) is presented, that allows to calibrate the complete frequency range in a short automated procedure. The vector network analyzer (VNA) and the corresponding electronically switched calibration (ECal) capabilities in combination with time domain gating and microwave network modelling are used to deembed the full system. The entire calibration requires not more than 5 minutes and the acquisition of one single electrostatic force microscopy (EFM) approach curve. In order to demonstrate the broadband capabilities, calibrated approach curves at various frequencies are presented. Nano Schottky diodes on a semiconductor substrate as well as biological cells were measured to demonstrate that the sample conductance and susceptance are in agreement with the theoretical expectations for the samples. This advanced workflow of quantitative impedance calibration may have many applications in the fields of semiconductor failure analysis, 2 dimensional materials and biological samples in their native liquid environment.
\end{abstract}

Index Terms-Scanning microwave microscopy, reflection calibration, ECal, time domain gating, de-embedding.

\section{INTRODUCTION}

Scanning Microwave Microscopy (SMM) combines the nanometric spatial resolution of an atomic force microscope (AFM) with the high electrical sensitivity of a vector network analyzer (VNA) at microwave frequencies. SMM allows to acquire both, mechanical and electrical images of the sample under study at the same time. The piezoelectric scanner moves the electrically conducting tip with nanometric tip radius (10 $\mathrm{nm}-1 \mu \mathrm{m})$ over the sample surface pixel by pixel while maintaining a constant contact force. The VNA sends an incident microwave signal towards the tip where it gets reflected partly. The reflected part is measured by the VNA's receiver and internally the ratio of reflected and incident signal, the $S_{11}$ scattering parameter, is calculated. This parameter contains information on the intrinsic electric and magnetic sample properties.

SMM has a wide range of applications in diverse areas like high and low loss material characterization [1], [2], semiconductor dopant concentration measurements, device

This work has been supported by EU FP7 (PEOPLE-2012-ITN-317116, NANOMICROWAVE), Bio-SMM FFG (Project No. 846532), and FWF Project P28018-B27.

M. Kasper and F. Kienberger are with Keysight Technologies Austria GmbH, Keysight Labs, Gruberstrasse 40, 4020 Linz, Austria (e-mail: manuel.kasper@keysight.com).

G. Gramse is with the Biophysics Institute, Johannes Kepler University of Linz, Gruberstrasse 40, 4020 Linz, Austria. failure analysis [3] - [6] and measurements on biological samples [7] - [9]. Application to minerals characterization has been reported [10] and subsurface measurement capabilities have been shown [11], [12]. Interferometric hardware improvements are presented in [13] and reviewed in [14]. In contrast to Scanning Capacitance Microscopy (SCM) SMM works broadband at significantly higher frequencies of 1-20 $\mathrm{GHz}$ and can measure not only capacitance but also resistive material properties.

For quantitative SMM measurements a fundamental step is the electrical "calibration" of the transmission line delivering the electrical stimulus to the SMM probe. The final aim of the calibration procedure is to obtain from the $S_{11}$ measurements quantitative complex admittance/impedance values of the nanoscale sample under study. A few calibration procedures were proposed [15], [16]. Typically, these procedures require defined capacitances, for example nanoscopic physical capacitors on a dedicated calibration sample. Alternatively, the capacitance variation when approaching the SMM probe towards the sample can be used for calibration at a given frequency by employing additional techniques [17], [18]. Time domain transformation has been shown to be a useful technique for SMM [19].

The workflow proposed here has the big advantage that it calibrates the SMM response in the entire frequency range at once requiring only one approach curve and two frequency sweeps. The basic idea is to shift the coaxial calibration plane as far as possible towards the AFM probe. This is achieved by a combination of traditional microwave techniques, however, without the use of the common coaxial calibration standards which cannot be connected to the system. With the calibration plane directly at the tip, the reflection is directly related with the complex tip impedance or admittance as will be shown. The workflow has been introduced for the first time in [20] where semi-quantitative results on a semiconductor sample were presented. In contrast, we present here a more advanced version leading to fully quantitative complex admittance/impedance measurements in a broad frequency range. Additional approach curves and images are shown in order to demonstrate the workflow.

The paper is organized as follows. In chapter two we present the theory and the experimental setup. The proposed calibration workflow consists of three steps which are discussed in the following three subsections. In subsection A we discuss the one-port VNA calibration with the ECal module and the corresponding de-embedding procedure while in subsection $\mathrm{B}$ the determination of a model of the matching 
network by use of time domain techniques is discussed. In subsection $\mathrm{C}$ we present a method for removing the remaining scaling error. In chapter three we present measured results. We apply the proposed calibration workflow to semiconductor samples and biological samples and the corresponding results are shown in subsection $\mathrm{A}$ and $\mathrm{B}$, respectively.

\section{CALIBRATION WORKFLOW}

The workflow described in this paper enables complete broadband calibration with only one known tip capacitance and is divided into three steps, which will be explained here further detailed in the below subsections.

In Fig. 1(a) the setup of the microwave components is shown. The AFM tip is connected to one side of the ECal module through a coaxial resonator cable at position 1 . The resonator cable in combination with the ECal module accounts for the impedance matching while the ECal module contains solid state switches which either select one of various impedances for reflection calibration of the VNA or the "thru" state which connects the VNA to the resonator cable. The second side of the ECal module is connected to the VNA by a connection cable at position 0 . The local tip-sample admittance

$$
\mathrm{Y}_{t i p}=G_{t i p}+j \omega C_{t i p}
$$

is the physical quantity of interest in the experiments where $G_{t i p}$ is the conductivity, $C_{t i p}$ the capacitance and $\omega$ the angular frequency of the $\mathrm{GHz}$-signal.

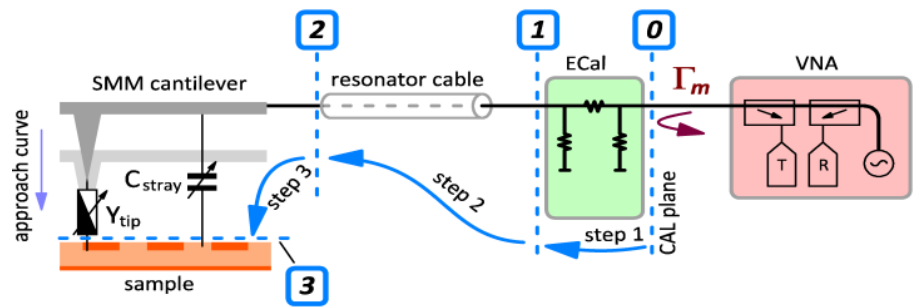

a)

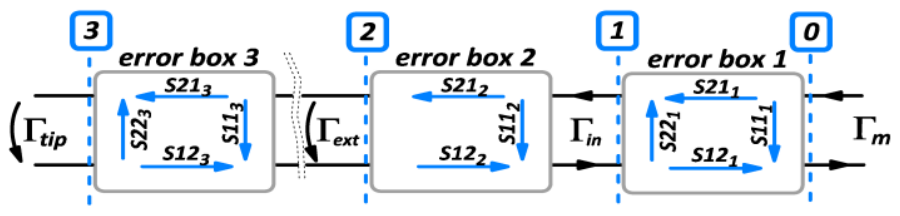

b)

Fig. 1. (a) Experimental setup with VNA, ECal, resonator cable, and nanometric SMM probe above the sample. Calibration moves calibration plane (dashed) successively towards the SMM probe. b) To extract $\Gamma_{\text {tip }}$ three error boxes corresponding to $\mathrm{ECal}$ (error box 1), resonator cable (2), and tip scaling effects (3) have to be de-embedded.
Fig. 1(a) also outlines the concept in which the calibration plane is successively shifted towards the AFM tip.

In a preparative step the VNA needs to be calibrated. This is done by the VNA's built-in one-port calibration procedure in combination with the ECal module. The calibration plane is then at position 0 . In order to shift the calibration plane to the next positions de-embedding techniques are used which allow to remove mathematically the effect of two-port networks from the measured result. First, to measure $S_{11}$ at position 1 the ECal module is switched in the "thru" state and the corresponding two-port network is de-embedded. The second step is to de-embed a two-port network that represents the resonator cable or at least a good approximation of it. In order to create this model, time-domain techniques are used. After this step the calibration plane has moved to position 2 and the measured reflection coefficient now already represents the tip impedance only distorted by a frequency dependent scaling error and an offset. The offset can be removed by relating the measurement to a reference position while the scaling error is cured by a simple third step based on one capacitance value measured by electrostatic force microscopy (EFM). It should be noted that the procedure covers the complete frequency range of operation $(1 \mathrm{GHz}-20 \mathrm{GHz})$ at once.

In Fig. 1(b) the concept of cascaded error boxes is introduced. The positions of the calibration planes correspond to that in Fig 1(a). The term "error box" is used in this paper in a general sense for a two-port network that needs to be deembedded. Each arrow in the error boxes corresponds to one frequency dependent complex coefficient that is determined during the calibration procedure.

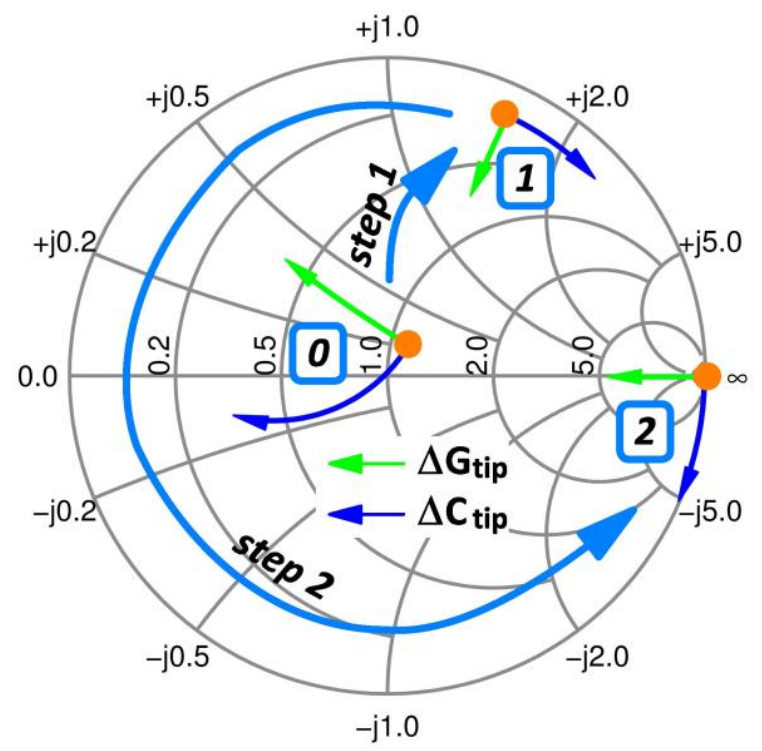

Fig. 2. The multi-step calibration procedure shown in the Smith Chart with the changes in tip conductance and capacitance indicated by straight and curved arrows, respectively.

Fig. 2 shows $S_{11}$ for the calibration steps one and two for a single frequency in the Smith chart. Typically, the SMM 
working frequency is selected such that the reflection coefficient is low corresponding to a good match. This allows accurate measurements at Pos. 0 in Fig. 2. For a higher tip conductance the working point moves on a straight line while an increasing tip capacitance results in a circularly shaped trajectory.

After de-embedding the ECal, which is an essential part of the resonant matching, (step 1) the resonant behavior is mathematically removed and what is left is the response of the resonator cable with the tip at its end (Pos. 1). The tip admittance is typically very low which corresponds to a tip impedance that is very high in comparison to the system impedance of $50 \Omega$. In addition, the resonator cable has low loss and therefore the system appears now almost entirely reflective. Calibration step 2 de-embeds a two-port network which represents the resonator cable. During this step the tip is placed at a position which is called reference position (e.g., $3 \mu \mathrm{m}$ above the sample surface) and where both the capacitance and conductance reading will be zero after the calibration, respectively. After step 2 the measured reflection coefficient shows an "open" response in the full frequency range corresponding to Pos. 2 in Fig. 2.

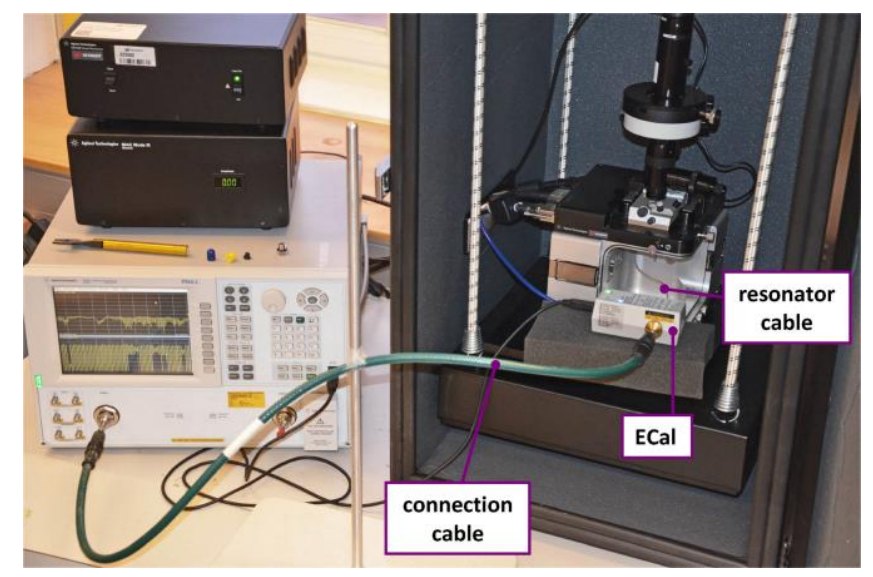

Fig. 3. Photograph showing the experimental setup. The ECal module is placed directly in front of the microscope.

The remaining step 3 has only very subtle effect on the reflection coefficient and is discussed later. It is important to understand that the calibration procedure does not influence the resonant impedance matching and the resulting SNR since it is a purely mathematical transformation. This also means that for initial tuning the calibration needs to be switched off otherwise the notches would be hidden.

Fig. 3 shows the experimental setup consisting of a Keysight N5230 VNA, a Keysight N4691 ECal module, and a Keysight 7500 SMM. The ECal module is placed closely to the SMM Nose Cone with the resonator cable directly attached. The VNA is connected to the second port of the ECal by means of a connection cable.

\section{A. VNA Reflection Calibration and De-embedding of the ECal Module}

For proper calibration of $S_{11}$ reflection measurements, typically three calibration standards are connected to a VNA's port in subsequence. Today, very reproducible electronically switchable calibration standards (ECal) are available which allow calibration with one single connection [21]. As shown in Fig. 1(a) one side of the ECal is connected to VNA by means of a connection cable. The VNA's internal calibration routine and the ECal are used to move the calibration plane to Pos. 0 (cf. Fig.1) eliminating all systematic VNA errors and connection cable effects. The frequency range is set to $1 \mathrm{GHz}$ to $20 \mathrm{GHz}$ and the number of points is set to 20001. Both settings remain unchanged for the complete workflow. Next, the ECal is switched into "thru" state connecting VNA and resonator cable. Fig. 4 shows the measured reflection coefficient $\Gamma_{\mathrm{m}}$ with the ECal in the "thru" state at Pos. 1 for the uncalibrated case (solid trace) and for the case where the systematic VNA errors and the connection cable effects have been removed (dashed trace). Both traces show the typical resonant response coming from the impedance matching while the high frequent ripple coming from standing waves on the connection cable has disappeared for the calibrated case.

As stated before, a resonant type of SNR improvement is used in the shown SMM architecture and the ECal module plays a key role in order to enable this resonant approach to work. In previous SMM implementations a fixed $50 \Omega$ shunt resistor was installed instead of the ECal [15] - [17]. Effectively being in parallel with the $50 \Omega$ resonator cable the shunt resistor forms a significant mismatch. Only at frequencies where the actual wavelength corresponds to an integer number of half of the electrical length of the resonator cable the system becomes well matched. At these frequencies, which are typically used for SMM measurements, the SNR becomes very good allowing sensitive measurements. In the frequency domain the magnitude of the reflection coefficient shows a periodic pattern with the notches corresponding to the resonant matching frequencies (cf. Fig 4). From a calibration perspective the shunt resistor is a problem because it has a hard to determine effect on the measured reflection coefficient. Instead we use the "thru" state of the ECal which firstly is not completely free of loss and secondly also not perfectly matched. Therefore, it also adds a significant discontinuity, just like the shunt resistor. In fact, the exact properties of this discontinuity are not of special importance and we noticed no degradation of the SNR compared to the shunt approach. The big advantage with ECal is, however, that the discontinuity is known exactly as it is a calibration standard with a Touchstone standard definition file. For calibration step 1 the VNA's two-port de-embedding function is used to de-embed this file which effectively shifts the calibration plane to Pos. 1 (cf. Fig. 1). 


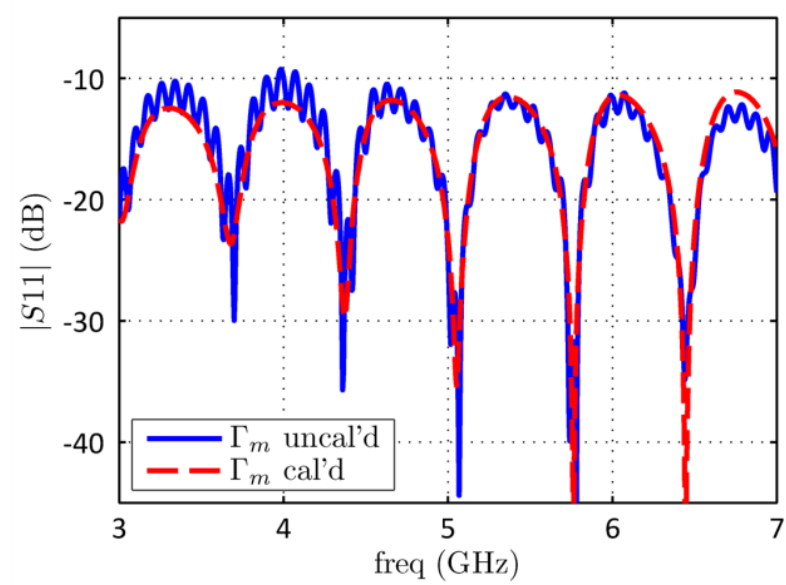

Fig. 4. Measured reflection coefficient $\Gamma_{\mathrm{m}}$ as a function of frequency before (solid) and after (dashed) reflection calibration with the ECal set to "thru". Systematic VNA errors and the effect of the connection cable cause significant ripple which is removed after this preparative calibration step.

\section{B. De-embedding of the Resonator Cable}

Calibration step 2 removes the effect of the resonator cable and moves the working point to the "open" point. This is done by determining a two-port model of the resonator cable and subsequent de-embedding. For this step the tip is placed $3 \mu \mathrm{m}$ above the sample surface which is the reference position to which all subsequent tip capacitance and tip conductance changes are referred. Error box 2 in Fig. 1(b) shows how the model looks like. The reflection $S_{11,2}$ summarizes all unwanted reflections that are not directly coming from the tip while $S_{21,2}$ and $S_{12,2}$ are transmission coefficients which represent the electrical length of the resonator cable. Since the resonator cable is reciprocal $S_{21,2}=S_{12,2}$.

Figure 5(b) shows $\Gamma_{\text {in }}$ in the frequency domain (solid trace). The typical response of an open ended lossy transmission line in combination with some ripple can be observed. The response is then transformed into the time domain by the VNA's internal capabilities. Additional information is provided in [22]. The result is shown in linear units in Fig. $5(\mathrm{a})$. The prominent signal at $3 \mathrm{~ns}$ is the main reflection coming from the tip. This reflection is slightly changing when the tip admittance is changing. At $100 \mathrm{ps}$ a much smaller reflection, which is related with the transition from the SMAconnector to the resonator cable, can be identified. This is an unwanted signal which causes ripple in the frequency domain. Similarly, at $5.8 \mathrm{~ns}$ there is a second order reflection which is reflected twice by the tip and once by the connector transition. Further higher order reflections can be found, however, their contribution is insignificant. Theoretically the second order reflection is also influenced by changes of the tip admittance but we observed that the simplification of treating it as constant does not have any negative effect on the result.

The aim of the time domain step is to separate the main reflection from the unwanted signals. This is done by applying a gate function which is shown in Fig. 5(a) (dashed box). The gate removes the main reflection and what is left is the unwanted part. Since these unwanted signals are with good approximation independent from the tip admittance, the transformation and the gating need to be done only once during the calibration. This is in contrast to [19] where the time domain procedures were applied on the individually measured frequency domain data of each pixel during imaging. The gated response is transformed back into the frequency domain (cf. Fig. 4(b), dotted trace) and is directly used as $S 11_{2}$ in error box 2. In order to de-embed series connected error boxes, the $S$-parameter network data of each box is converted into ABCD-parameters using

$$
\begin{array}{ll}
A=\frac{\left(1+S_{11}\right)\left(1-S_{22}\right)+S_{12} S_{21}}{2 S_{21}} & B=Z_{0} \frac{\left(1+S_{11}\right)\left(1+S_{22}\right)-S_{12} S_{21}}{2 S_{21}} \\
C=\frac{1}{Z_{0}} \frac{\left(1-S_{11}\right)\left(1-S_{22}\right)-S_{12} S_{21}}{2 S_{21}} & D=\frac{\left(1-S_{11}\right)\left(1+S_{22}\right)+S_{12} S_{21}}{2 S_{21}}
\end{array}
$$

ABCD-parameters easily allow to calculate the parameters of series connected networks by multiplying the associated ABCD-parameter matrices. The result is then transformed back into $S$-parameters and written in a file which can be directly used by the VNA's de-embedding function. In order to obtain the correct $S_{21,2}$ and $S_{12,2}$ temporarily both, $S_{21,2}$ and $S_{12,2}$ are set to one. After that, the first two error boxes are combined and the result is de-embedded. When $S_{11}$ is measured now, the result looks like the dashed trace shown in Fig. 5(b). Without the ripple the result looks like a transmission line response with some loss and constant group delay (not shown) representing the resonator cable and the tip. Since the tip is placed in air it can be assumed that the signal gets fully reflected by the tip which is expressed by $\Gamma_{e x t}=1$ in the signal flow graph in Fig. 1(b). This assumption allows to map the measured response equally to $S_{21,2}$ and $S_{12,2}$ resulting in a fully determined error box 2 which is finally de-embedded instead of the temporarily defined one. The reflection coefficient of the system is equal to an "Open" response for all frequencies after this process (cf. Fig. 1 Pos. 2). 


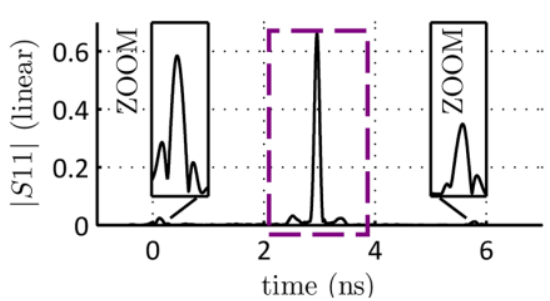

a)

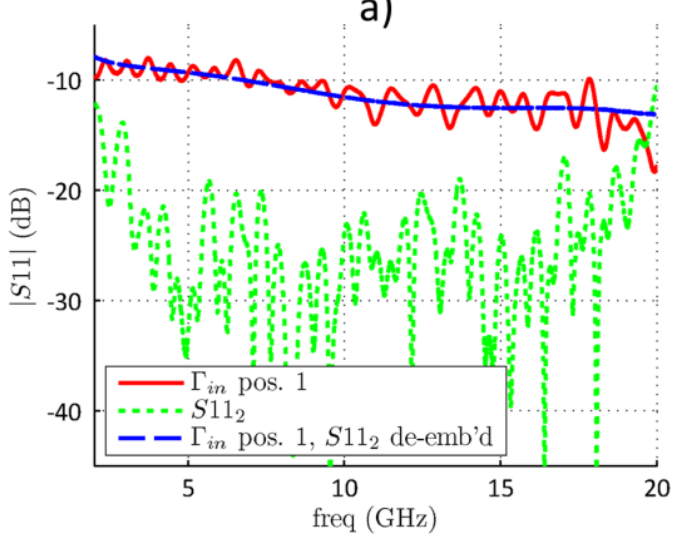

b)

Fig. 5. (a) Time domain transformed system reflection coefficient. The first box shows small reflections from the ECal discontinuity. The second box represents the SMM tip which is removed by the gate (dashed). (b) Magnitude of system reflection coefficient after calibration step 1 in the frequency domain. The solid trace includes the tip reflection and all discontinuities while the dotted trace shows only the discontinuities with removed (gated) tip reflection. Finally, the dashed trace shows the system response after de-embedding the discontinuities.

\section{Response Normalization}

As explained before, the reflection coefficient frequency response with the tip in the reference position shows a flat line at $0 \mathrm{~dB}$ for the amplitude and $0 \mathrm{deg}$. for the phase. When the tip is now placed at a different position (e.g., the sample surface) the capacitance and the corresponding phase have changed but in general there is still a frequency dependent scaling error on this phase change. The aim of calibration step 3 is to correct for this scaling error effectively moving the calibration plane to Pos. 3 (cf. Fig. 1). This step is based on acquiring one EFM approach curve to determine error box 3 (cf. Fig. 1 (b)). The electrostatic force, $F_{e s, 2 \omega}$ is related to the capacitance, $C(z)$ by

$$
\frac{d C}{d z}=\frac{4 F_{e s, 2 \omega}}{V_{0}^{2}}
$$

where

$$
v(t)=V_{0} * \sin (\omega t)
$$

is the excitation voltage. By integration, the capacitance with the tip in contact $C\left(z_{\text {contact }}\right)$ is obtained. This method is discussed extensively in [17]. With the tip in contact another frequency sweep is performed. Since the capacitance and the corresponding phase for the reference position $(z=3 \mu \mathrm{m})$ is defined to be zero and the capacitance and the phase at contact has been measured one intermediate point can be constructed by linear interpolation. The resulting three points and the relationships

$$
\begin{aligned}
& Z_{\text {tip }}=\frac{1}{j \omega C} \stackrel{\text { ields }}{\rightarrow} \Gamma_{t i p}=\frac{Z_{t i p}-Z_{0}}{Z_{t i p}+Z_{0}} \\
& \Gamma_{m}=S 11_{3}+\frac{S 21_{3} S 12_{3} \Gamma_{t i p}}{1-S 22_{3} \Gamma_{t i p}}
\end{aligned}
$$

are used to obtain the coefficients $S_{11,3}, S_{21,3} * S_{12,3}$ and $S_{22,3}$ where $Z_{0}=50 \Omega$ is the characteristic system impedance. The approach of determining another error box has been chosen because it nicely integrates with the previous steps leading to one final Touchstone file for the de-embedding process. However, an alternative would be to directly scale the measured capacitance and conductance values and omit error box 3 . In contrast to [17], the presented calibration workflow is mainly relying on the ECal calibration standard while the single approach curve acquired is only used to obtain a response correction. It is important to note that without the preceding steps one approach curve would be required for each individual frequency.

\section{RESULTS}

For calibration steps one and two that run in an automated procedure, the tip has been placed $3 \mu \mathrm{m}$ above the sample surface. After that, one single EFM approach curve $(3 \mu \mathrm{m}$ distance) is acquired. The measured capacitance difference $\Delta C=480 \mathrm{aF}$ is used to calculate error box 3 and to scale the response of the system at all frequencies. In order to prove that the calibration workflow is broadband and leaves the system calibrated for the entire range of frequencies, in Fig. 6 additional approach curves were performed on the doped silicon substrate at various frequencies. As can be seen the calibrated SMM approach curves show a consistent capacitance for all frequencies. Similarly, the conductance shows only very little excursion as expected. This proves that the calibration has been successful over the entire frequency range.

Interestingly, we observed that the approach curves acquired at low frequencies $(<3.2 \mathrm{GHz})$ did not change from calibration step 2 to 3 and were already scaled correctly after calibration step 2. This fact opens the option to perform the entire calibration without the need of any additional technique like EFM. However, this point has to be studied more carefully and will be subject of further investigations. 

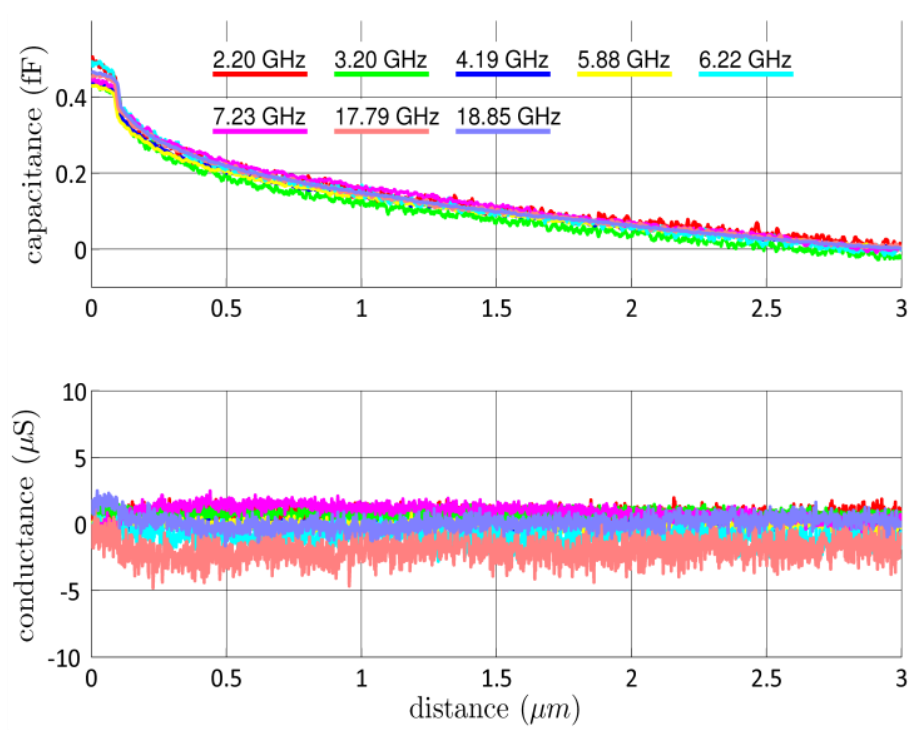

Fig. 6. SMM approach curves at different frequencies after calibration step 3. Both, capacitance (top) and conductance (bottom) show basically the same response for all measured frequencies.

\section{A. Semiconductor Samples}

SMM images of a semiconductor test sample were acquired (Fig. 7). The sample consists of different MOS capacitors placed over various oxide layers with different thicknesses ranging from 50 to $200 \mathrm{~nm}$. Gold pads without any oxide below are forming Schottky diodes. The diameter of the MOS and Schottky gold pads ranges from 1-4 $\mu \mathrm{m}$. The calibrated capacitance of the gold pads ranges from $100 \mathrm{aF}$ to $9 \mathrm{fF}$ (Fig $7 b$ ) in accordance to previous studies [23]. Fig. 7(b) shows the calibrated complex tip admittance for reverse bias including the conductivity image in milli-Siemens and the capacitance image in femto-Farad. The conductance image shows no conductivity both for MOS and Schottky gold pads. Variable capacitor properties are obtained in the capacitance image for the Schottky pads whereas MOS pads have constant capacitance. Forward bias results in high conductance values of the Schottky pads with values in the range of $0.1 \mathrm{mS}-$ $1 \mathrm{mS}$ while the MOS pads show no significant signal (Fig. 7(c)). The capacitance of the MOS capacitors is, depending on the gold pad surface area and the dielectric oxide thickness, well within the expected range of a parallel plate capacitor, with the forward biased Schottky diodes showing similarly high capacitances.

\section{B. Biological Samples}

Biological samples were investigated with the proposed method. Fig. 8 shows the calibrated measurement of ARPE-19 (Arising Retinal Pigmented Epithelia) cells at $8.42 \mathrm{GHz}$. The cells were dried and chemically fixed on a conductive silicon substrate with the measurement done in air. In Fig. 8(a) the topographical image is shown at a scan size of $36 \mathrm{um}$ x $36 \mathrm{um}$. The topographical measurement was corrected for sample tilting by subtracting a linear plane.

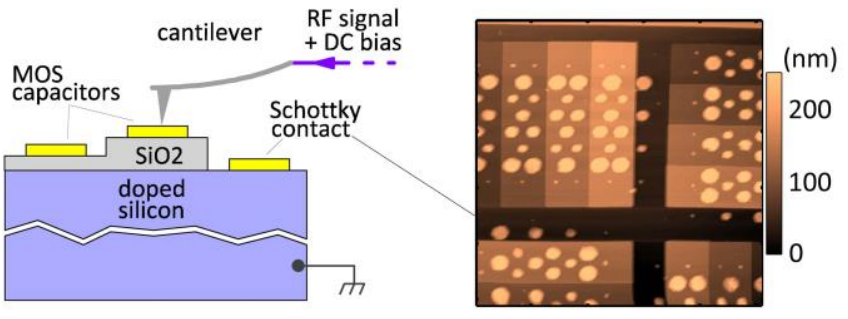

a)
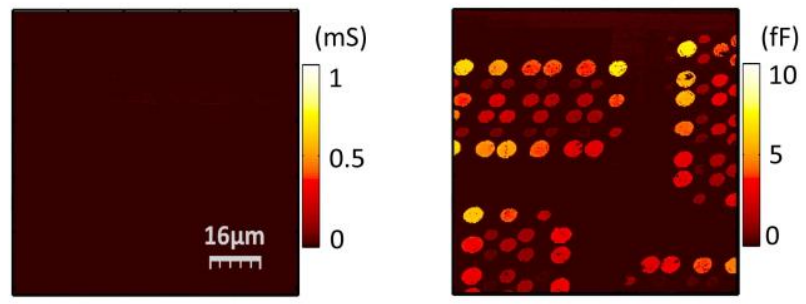

b)
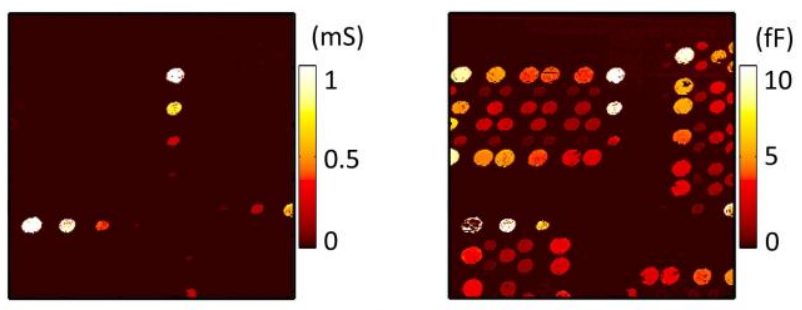

c)

Fig. 7. (a) Sketch of the gold pad sample (left). Both MOS capacitors and Schottky diodes are represented, with $\mathrm{SiO} 2$ and without, respectively. The topographical image (right) is shown with the color bar giving the height of the sample (ranging from 0-200 nm). The lateral image size is $80 \mu \mathrm{m}^{2}$. (b) Calibrated parallel conductance (in units of milli-Siemens) and capacitance data (in units of femto-Farad) for the various gold MOS and Schottky pads in reverse bias. (c) In forward bias the Schottky diodes show conductive lossy behavior. SMM frequency was $6 \mathrm{GHz}$.

The overall cell height is up to $1 \mu \mathrm{m}$ while the lateral cell diameter is roughly $5 \mu \mathrm{m}-7 \mu \mathrm{m}$. In Fig. 8(b) the corresponding SMM conductance and capacitance images are shown. In the conductance image there is almost no signal difference between cell and substrate indicating low losses of the dried cells. Since water is mostly removed due to the drying process and the imaging in air, also the corresponding conductivity coming from water within the cell and surrounding the cell is reduced. This is in line with previous SMM measurements done at different humidity levels [8]. The capacitance image shows a strong contrast. The values are relative with respect to the substrate. As shown in a recent study [9], variations in the cell capacitance are typically coming from different dielectric behavior of cell compartments with different chemical composition and density. As such the calibrated capacitance image is sensitive to biologically relevant information of the cell interior. 


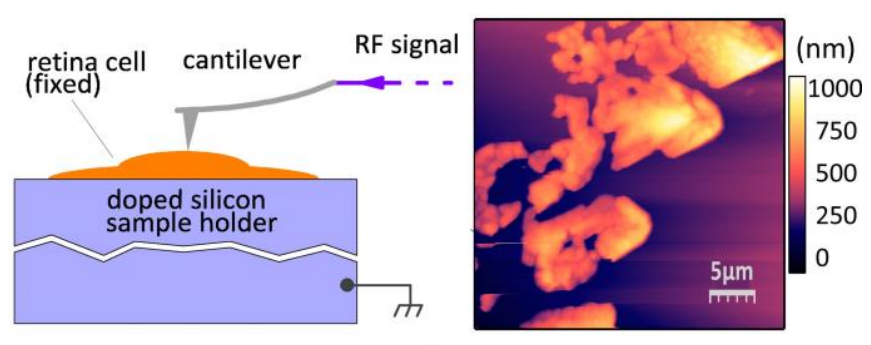

a)
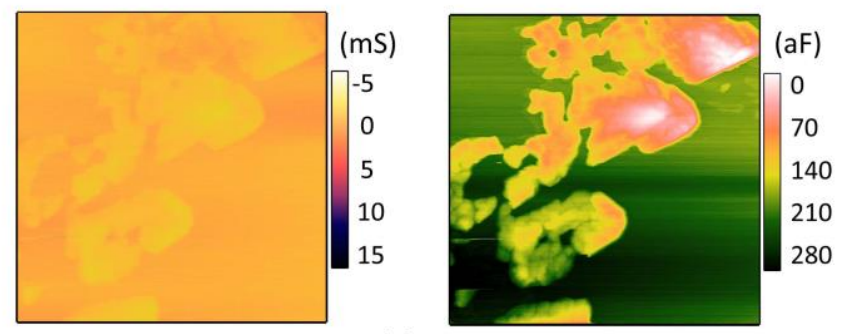

b)

Fig. 8. (a) Sketch of biological cell measurements in air (left). ARPE19 cells are placed on a conductive silicon substrate and are imaged with the SMM. The topography is shown (right) with a scan size of $36 \mu \mathrm{m} \times 36 \mu \mathrm{m}$. (b) Calibrated conductance and capacitance after calibration step 3 measured at $8.42 \mathrm{GHz}$. The conductance image shows almost no contrast indicating low losses in the dried and chemically fixed cells. The capacitance image shows strong contrast corresponding to variations dielectric properties.

\section{CONCLUSION}

A calibration workflow for SMM was presented which splits up the complex $S_{11}$ reflection coefficient accurately into conductive and capacitive response, without the need of nanoscale calibration samples and valid for the full span of 1$20 \mathrm{GHz}$ frequencies. Only one capacitance difference value, which we obtain from an EFM approach curve, has to be known. After this calibration the frequency can be easily changed during SMM imaging without losing the calibration. With this new calibration various materials can be quantitatively studied at different frequencies, including dopant profiling of semiconductors where frequency dependent measurements are relevant. Also various applications can be considered for subsurface imaging where the electromagnetic field of the SMM tip penetrates through the material with the immersion depth adjustable by the SMM operating frequency. The new method is expected to have applications for semiconductor dopant calibrations, advanced materials science measurements, and accurate dielectric capacitance measurements of biological samples including bacteria and living cells. The calibration workflow presented here allows to determine more accurately the doping density and depletion layer capacitance in semiconductor samples, the conductivity of advanced materials including graphene, and the dielectric properties of cell compartments and biomolecular water hydration at broadband $1-20 \mathrm{GHz}$ frequencies.

\section{ACKNOWLEDGEMENT}

The authors would like to thank Lilia Chtcheglova and Silviu Tuca from Johannes Kepler University of Linz for cell sample preparation.

\section{REFERENCES}

[1] A. Gregory, J. F. Blackburn, K. Lees, R. N. Clarke, T. E. Hodgetts, S. M. Hanham et al., "Measurement of the permittivity and loss of high-loss materials using a Near-Field Scanning Microwave Microscope," Ultramicroscopy, vol. 161, pp. 137-145, Feb. 2016.

[2] J. Hoffmann, G. Gramse, J. Niegemann, M. Zeier, and F. Kienberger, "Measuring low loss dielectric substrates with scanning probe microscopes," Appl. Physics Lett., vol. 105, no. 1, pp. 013102-013106, July 2014.

[3] E. Brinciotti, G. Campagnaro, G. Badino, M. Kasper, G. Gramse, S. S. Tuca et al, "Frequency Analysis of Dopant Profiling and Capacitance Spectroscopy Using Scanning Microwave Microscopy," IEEE Trans. Nanotechnol., vol. 16, no. 1, pp. 75-82, Jan. 2017.

[4] E. Brinciotti, G. Gramse, S. Hommel, T. Schweinboeck, A. Altes, M. Fenner et al., "Probing resistivity and doping concentration of semiconductors at the nanoscale using scanning microwave microscopy," Nanoscale, vol. 7, no. 35, pp. 1471514722, Sept. 2015.

[5] A. Imtiaz, T. M. Wallis, S. H. Lim, H. Tanbakuchi, H. P. Huber, A. Hornung et al., "Frequency-selective contrast on variably doped p-type silicon with a scanning microwave microscope," $J$. Appl. Phys., vol. 111, no. 9, pp. 093727-093733, May 2012.

[6] H. P. Huber, I. Humer, M. Hochleitner, M. Fenner, M. Moertelmaier, C. Rankl et al., "Calibrated nanoscale dopant profiling using a scanning microwave microscope," J. Appl. Phys., vol. 111, no. 1, pp. 014301-014310, Jan. 2012.

[7] Y. J. Oh, H. P. Huber, M. Hochleitner, M. Duman, B. Bozna, M. Kastner et al., "High-frequency electromagnetic dynamics properties of THP1 cells using scanning microwave microscopy," Ultramicroscopy, vol. 111, pp. 1625-1629, Nov. 2011.

[8] S. S. Tuca, G. Badino, G. Gramse, E. Brinciotti, M. Kasper, Y. J. Oh et al., "Calibrated complex impedance of $\mathrm{CHO}$ cells and $\mathrm{E}$ coli bacteria at $\mathrm{GHz}$ frequencies using scanning microwave microscopy," Nanotechnology, vol. 27, no. 13, pp. 135702135711, Apr. 2016.

[9] M. C. Biagi, R. Fabregas, G. Gramse, M. Van Der Hofstadt, A. Juarez, F. Kienberger et al., "Nanoscale Electric Permittivity of Single Bacterial Cells at Gigahertz Frequencies by Scanning Microwave Microscopy," ACS Nano, vol. 10, no. 1, pp. 280-288, Jan. 2016.

[10] T. Monti, A. Tselev, O. Udoudo, I. N. Ivanov, C. Dodds, and S. W. Kingman, "High-resolution dielectric characterization of minerals: A step towards understanding the basic interactions between microwaves and rocks," Int. J. Mineral Proc., vol. 151, pp. 8-21, Jun. 2016.

[11] G. Gramse, E. Brinciotti, A. Lucibello, S. B. Patil, M. Kasper, C. Rankl et al., "Quantitative sub-surface and non-contact imaging using scanning microwave microscopy," Nanotechnology, vol. 26, no. 13, pp. 135701-135710, Apr. 2015.

[12] C. Plassard, E. Bourillot, J. Rossignol, Y. Lacroute, E. Lepleux, L. Pacheco et al., "Detection of defects buried in metallic samples by scanning microwave microscopy," Physical Review, vol. 83, no. 11, pp. 121409-121413, Mar. 2011. 
[13] T. Dargent, K. Haddadi, T. Lasri, N. Clément, D. Ducatteau, B. Legrand etal., "An interferometric scanning microwave microscope and calibration method for sub-fF microwave measurements," Rev. Sci. Instrum., vol. 84, no. 12, pp. 123705123712, Dec. 2013.

[14] A. Imtiaz, T. M. Wallis, and P. Kabos, "Near-field scanning microwave microscopy: An Emerging Research Tool for Nanoscale Metrology," IEEE Microw. Mag., vol. 15, no. 1, pp. 52-64, Jan. 2014.

[15] J. Hoffmann, M. Wollensack, M. Zeier, J. Neigemann, H.-P. Huber, and F. Kienberger, "A Calibration Algorithm for Nearfield Scanning Microwave Microscopes," in 12th IEEE Int. Conf. Nanotechnology, Birmingham, 2012, pp. 1-4.

[16] H. P. Huber, M. Moertelmaier, T. M. Wallis, C. J. Chiang, M. Hochleitner, A. Imtiaz et al., "Calibrated nanoscale capacitance measurements using a scanning microwave microscope," Rev. Sci. Instrum., vol. 81, no. 11, pp. 113701-113710, Nov. 2010.

[17] G. Gramse, M. Kasper, L. Fumagalli, G. Gomila, P. Hinterdorfer, and F. Kienberger, "Calibrated complex impedance an permittivity measurements with scanning microwave microscopy," Nanotechnology, vol. 25, no. 14, pp. 145703-145711, Apr. 2014.

[18] M. Farina, D. Mencarelli, A. di Donato, G. Venanzoni, and A. Morini, "Calibration Protocol for Broadband Near-Field Microwave Microscopy," IEEE Trans. Microw. Theory Tech., vol. 59, no. 10, pp. 2769-2776, Oct. 2011.

[19] M. Farina, A. Lucesoli, T. Pietrangelo, A. di Donato, S. Fabiani, G. Venanzoni et al., "Disentangling time in a near-field approach to scanning probe microscopy," Nanoscale, vol. 3, no. 9, pp. 3583-3593, Aug. 2011.

[20] M. Kasper, G. Gramse, and F. Kienberger, "An advanced impedance calibration method for nanoscale microwave imaging," in 2016 IEEE MTT-S Int. Microwave Symp. (IMS), San Francisco, CA, Aug. 2016, pp. 1-4.

[21] A. Ferrero, "Two-port network analyzer calibration," in Modern RF and Microwave Measurement Techniques, V. Teppati, A. Ferrero, and M. Sayed, Eds., $1^{\text {st }}$ ed. Cambridge, UK: Cambridge University Press, 2013, ch. 8, pp. 195-217.

[22] J. P. Dunsmore, "Time Domain Transforms," in Handbook of Microwave Component Measurements with Advanced VNA Techniques, $1^{\text {st }}$ ed. New York: Wiley, 2012, ch. 4, pp. 211-242.

[23] M. Kasper, G. Gramse, J. Hoffmann, C. Gaquiere, R. Feger, A. Stelzer et al., "Metal-oxide-semiconductor capacitors and Schottky diodes studied with scanning microwave microscopy at $18 \mathrm{GHz}, " J$. Appl. Phys., vol. 116, no. 18, pp. 184301-184309, Nov. 2014. 